\title{
Nanomechanical Testing: Challenges and Opportunities
}

\author{
Xiaodong Li and Xinghang Zhang
}

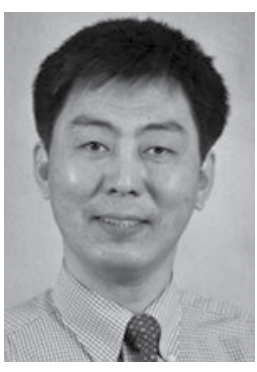

Xiaodong Li

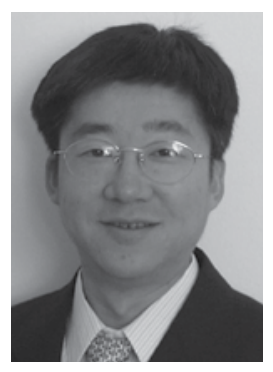

Xinghang Zhang

Recent developments in science and engineering have enriched our capability to fabricate and control structures, materials, and devices on the scale of nanometers. The functionality and/or reliability of micro/nanodevices are often determined by the mechanical properties of individual nanostructures used to construct the devices. It has been shown that mechanical properties of materials are size-dependent. The mechanical property values of bulk materials cannot be used for designing and manufacturing micro/nanodevices. Most importantly, the size-dependent deformation mechanisms and mechanical properties of nanostructures are not well understood. Such knowledge is critical for the further development of high-quality micro/nanodevices.

The extremely small dimensions of nanostructures (for instance, nanoparticles, nanotubes, and nanowires, to name a few) have imposed great challenges to many existing instruments, methodologies, and even theories. Tensile testing requires that the sample size be sufficiently large to be clamped rigidly without sliding. This is almost impossible for nanostructures such as nanowires. Furthermore, the extremely small size of nanostructures makes their manipulation rather difficult and specialized instruments and techniques are needed for the selection and assembly of individual nanostructures. On the other hand, calibration procedures for nanomechanical testing have been largely ignored, consequently resulting in some controversial results in the literature.

Bulk nanostructured materials (nanocrystalline materials) in which grain size is on the order of $100 \mathrm{~nm}$ or less provide a new platform for studying the effects of grain boundaries, twins, and dislocations on their deformation behavior and mechanical properties. For example, a reverse Hall-Petch effect has been observed in some metallic nanocrystalline materials.

Recentadvances in scanning electron microscopy (SEM) techniques enable us to observe microstructures at a few nanometers resolution. J.R. Greer et al. present state-of-the-art in-situ SEM and transmission electron microscopy (TEM) nanomechanical testing techniques with the article titled "The Insitu Mechanical Testing of Nanoscale Single-Crystalline Nanopillars." A nanomechanical tester was integrated with an SEM to perform compression and tension tests on micro/nanopillars or dog-bone tensile bars in-situ where local, nanoscale changes in sample surface morphology such as slip lines were imaged/recorded simultaneously by the SEM. The article also describes the experimental procedures and calibration details which may help the development of other nanomechanical testing techniques. TEM allows us to "see" crystal structure and defects such as grain boundaries, dislocations, and twins which are impossible (or difficult) for SEM to image. To perform nanomechanical testing inside the TEM imposes new challenges and opportunities for materials scientists.

The nanoscale structure of pores and ligaments in bulk nanoporous materi- als poses difficulties for experimentally studying their deformation behavior and fracture mechanics. In the article titled "Tensile and Compressive Microspecimen Testing of Bulk Nanoporous Gold," Balk et al. demonstrate novel approaches to testing such nanostructured materials using a microspecimen. The digital image correlation technique was used to analyze local small-scale strain field and then used to calculate elastic modulus and Poission's ratio. The detailed fracture surface studies in conjunction with the analysis of corresponding stress-strain curves give us an in-depth understanding of the mechanical behavior of nanoporous materials.

Mechanical properties of solid surfaces and thin films at elevated temperature are of great interest since many thin-film based devices operate at high temperature or harsh environment. Z.C. Duan and A.M. Hodge review the current state and history of high-temperature nanoindentation capability with a focus on recent research topics and available testing systems with the article titled "High-Temperature Nanoindentation: New Developments and Ongoing Challenges." Their future outlook on this topic presents the urgent need for nanoindenter manufacturers to develop high-temperature capability, and may draw more attention to high-temperature properties of thin films.

Xiaodong $\mathrm{Li}$ is CEC Distinguished Professor in the Department of Mechanical Engineering at the University of South Carolina, Columbia, SC. Xinghang Zhang is an assistant professor in the Department of Mechanical Engineering, Materials Science and Engineering Program, at Texas A\&M University, College Station, TX. Li and Zhang are the advisors to JOM from the Nanomechanical Materials Behavior Committee of the Materials Processing \& Manufacturing Division of TMS. 